\title{
Ellagic Acid Protects against Activation of Microglia by Inhibiting MAPKs and NF-кB Signalling
}

\author{
Elysha Nur Ismail', Norazrina Azmi ${ }^{2, *}$, Ibrahim Jantan ${ }^{3}$ \\ ${ }^{1}$ Department of Biomedical Sciences, Faculty of Medicine and Health Sciences, Universiti Putra Malaysia, UPM Serdang, \\ Selangor, MALAYSIA. \\ ${ }^{2}$ Drug and Herbal Research Centre, Faculty of Pharmacy, Universiti Kebangsaan Malaysia, Jalan Raja Muda Abdul Aziz, \\ Kuala Lumpur, MALAYSIA. \\ ${ }^{3}$ Institute of Systems Biology (INBIOSIS), Universiti Kebangsaan Malaysia, UKM Bangi, Selangor, MALAYSIA.
}

\begin{abstract}
Context: One of the hallmarks of neurodegenerative diseases is dysregulation of microglia resulting in neuroinflammation. Neuroinflammation is related to multiple neurodegenerative disorders, including stroke and Alzheimer's disease. Ellagic acid (EA) possesses antiapoptotic activity, anti-inflammatory, and antioxidant properties. It is most commonly found in fruits and nuts. EA has been linked to neuronal protection particularly through its anti-inflammatory and antioxidant actions. For example, EA inhibits enzyme activity which prevents the onset of Alzheimer's disease. Aim: In this study, the underlying protective mechanisms of EA in LPS-activated BV2 cells were investigated. Settings and Design: EA prepared in multiple concentrations was introduced for 24 hrs as pre-treatment in BV2 microglial cells. Lipopolysaccharide (LPS) at $1 \mu \mathrm{g} \mathrm{mL}^{-1}$ was used to stimulate BV2 microglial cells after the EA pre-treatment for an additional $24 \mathrm{hrs}$. After $24 \mathrm{hrs}$ of stimulation, the cell lysate was harvested for bioassays. Materials and Methods: This study investigated the effects of EA on the viability of BV2 microglial cells using MTT assay. After LPS stimulation, the cell lysate was collected to measure nitric oxide (NO) levels using Griess assay kit and ELISA was used to measure TNFa levels. Western blotting was used to test protein levels of iNOS, MyD88, MAPKs and NF-kB. Statistical analysis used: Data are provided as means and standard errors of $n$ $=3$. A one-way analysis of variance was used to test the statistical significance of between group comparisons. The statistical significance of $p<0.05$ was set. Results: EA decreased the levels of TNFa and NO production in BV2 microglial cells induced by LPS. EA also reduced inducible nitric oxide synthase (iNOS) expression, and attenuated MyD88 and NF-kB expression. EA decreased the phosphorylation of p38, ERK and JNK and repressed the activation of $C D 11 \mathrm{~b}$ and $\mathrm{CD} 40$ in a concentration-dependent manner. Conclusion: EA has a putative role in preventing neuroinflammation but its application in prevention of neurodegenerative diseases requires further investigations.
\end{abstract}

Key words: Neuroinflammation, Anti-inflammation, Ellagic acid, Microglia, NF-kB, MAPKs.

Key Messages: The study indicated that ellagic acid may exert its antineuroinflammatory effects by inhibiting the development of MyD88, MAPKs and NF-kB signalling molecules.

\section{INTRODUCTION}

Altered inflammatory responses may ensue excessive damage to affected brain cells and will potentially lead to cell death and neurodegeneration. ${ }^{1}$ Microglia dysregulation has been linked to multiple neurodegenerative diseases such as dementia, ${ }^{2}$ amyotrophic lateral sclerosis, ${ }^{3}$ Parkinson's disease and Alzheimer's disease. ${ }^{4}$ As a central nervous system (CNS) resident cell, microglia control the central nervous tissue immune homeostasis. ${ }^{5}$ Microglia has been implicated in the role of both
Submission Date: 10-04-2020; Revision Date: 17-07-2020; Accepted Date: 07-09-2020

DOI: 10.5530/ijper.54.3s.152 Correspondence: Dr. Norazrina Azmi Drug and Herbal Research Centre, Faculty of Pharmacy, Universiti Kebangsaan Malaysia, Jalan Raja Muda Abdul Aziz-50300 Kuala Lumpur, MALAYSIA. Phone: +604-9289 7095 E-mail: azrina.azmi@ukm. edu.my

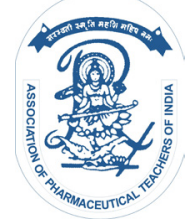

www.ijper.org 
causative and exacerbation in many neurodegenerative diseases and over-activation of microglia contributes to neuroinflammation. ${ }^{6}$ Chronically activated microglia due to brain injury, infections and inflammation, initiates a rapid inflammatory response that activates the development and release of massive amounts of pro-inflammatory cytokines (TNF $\alpha, \mathrm{IL}-1 \beta)$ and neurotoxic mediators (NO, COX-2), while increasing neurodegeneration and neuronal death.

There is an increasing awareness that inflammation is involved in the initial outcome of neurodegenerative disorders and in fact, inflammation-mediated neurodegeneration and microglia have also been implicated in other diseases and conditions such as stroke, ${ }^{8}$ hypoxia ${ }^{9}$ and neuropathic pain. ${ }^{10}$ Therefore, neuroinflammation has attracted attention for neuroprotective therapies in neurodegenerative disorders, particularly through inhibition of microglial activation. ${ }^{11}$ Bacterial component lipopolysaccharide (LPS) causes microglia to secrete various inflammatory molecules including tumour necrosis factor (TNF)- $\alpha$, interleukin (IL)-6, IL-1 $\beta$, nitric oxide (NO) and inducible nitric oxide synthase (iNOS) to promote inflammatory reactions. ${ }^{12}$ The inhibition of these mediators may thus also be a beneficial approach to neuroinflammatory prevention.

Experimental and epidemiological research is currently focused on the inhibitory impact of plant polyphenols on many peripheral inflammations including gastrointestinal diseases. Polyphenols can be used as additional anti-inflammatory agents or to complement non-steroidal anti-inflammatory drugs (NSAIDs) in the treatment of neuroinflammation. Ellagic acid (Figure 1) is a polyphenol typically found in berry and pomegranate fruits, vegetables and nuts. ${ }^{13}$ Various in vivo and in vitro studies have also shown that ellagic acid possesses pharmacological properties including anti-coagulant, ${ }^{14}$ anti-inflammatory, ${ }^{13-15}$ anti-haemorrhagic ${ }^{16}$ and antitumour effects. ${ }^{17}$ In this study, the neuroprotective effect of ellagic acid on LPS-mediated inflammatory response in murine BV2 microglial cells was investigated by determining the inhibition of NF-xB and MAPK signalling molecules.

\section{MATERIALS AND METHODS Cell culture}

The immortalized murine BV2 microglial cells were a gift from Associate Professor Dr. Sharmili Vidyadaran of the Immunology Laboratory, Faculty of Medicine and Health Sciences, Universiti Putra Malaysia. Cells were maintained in Dulbecco's modified Eagle's medium high glucose (Gibco, Grand Island, NY) complemented by foetal bovine serum (10\%; Nacalai, Japan), penicillin $\left(100 \mathrm{U} \mathrm{mL}^{-1}\right)$ and streptomycin $\left.100 \mu \mathrm{g} \mathrm{mL}^{-1}\right)$ at $37^{\circ} \mathrm{C}$ in a humidified atmosphere of $95 \%$ air, $5 \% \mathrm{CO}_{2}$. Confluent cultures were passaged by Accutase ${ }^{\mathrm{TM}}$ (Nacalai, Japan). Cell density was determined according to respective culture plates: $2.5 \times 10^{4}$ cells $/ \mathrm{cm}^{2}$ on 96-well, $5 \times 10^{4}$ cells $/ \mathrm{cm}^{2}$ on 24-well (NO measurement and ELISA) and $1 \times 10^{6}$ cells $/ \mathrm{cm}^{2}$ on 24 -well plates (flow cytometry and Western blot).

\section{Preparation of EA}

The lyophilised EA was purchased from Cayman Chemical, USA. EA powder was first weight and dissolved in 100\% DMSO (stock 1), sonicated for 10 mins and kept in $-80^{\circ} \mathrm{C}$. A second stock from stock 1 (now labelled as stock 2) was diluted in PBS, sonicated and kept in $-20^{\circ} \mathrm{C}$. Upon addition to the cells, stock 2 was dissolved in the culture medium accordingly to get the final concentration in the cell cultures.

\section{Preparation of LPS}

LPS was purchased from Sigma-Aldrich, St Louise, MT (strain from Escherichia coli 026: B6). A $1 \mu \mathrm{g} \mathrm{mL}-1$ of lyophilised LPS was weighed and dissolved in PBS, sonicated and kept in $-20^{\circ} \mathrm{C}$. Upon addition to cells, the LPS stock solution was dissolved in the culture medium accordingly to get the final concentration in the cell cultures.

\section{Treatment of cells}

Cells were pre-treated with EA $(0.5,1,5$ and $10 \mu \mathrm{M})$ for $24 \mathrm{hrs}$, then stimulated with LPS for another $24 \mathrm{hrs}$. Cell supernatant/lysate was collected after $24 \mathrm{hrs}$.

\section{Cell viability assay}

After cell treatment, culture media was discarded and MTT (0.5 $\mathrm{mg} \mathrm{mL}^{-1}$; Nacalai, Japan) was applied for $2 \mathrm{hrs}$ incubated at $37^{\circ} \mathrm{C}$. After discarding the cell supernatant, dimethyl sulfoxide (Sigma-Aldrich) was added to the wells. The developed crystals were mixed to dissolve and the plates were read on an automated absorbance reader, NanoQuant (Tecan Trading AG, Switzerland), using a $570 \mathrm{~nm}$ and a reference of $630 \mathrm{~nm}$ wavelength.

\section{Nitrite measurement}

Secreted NO amounts were measured using the Griess reagent (Sigma-Aldrich). Supernatants of the treated cells were mixed with Griess reagent (of equal volume) in a 96-well plate and allowed to stand at room temperature for $10 \mathrm{~min}$. Nitrite concentrations were determined following instructions by manufacturer at a wavelength of $540 \mathrm{~nm}$. 


\section{Enzyme-linked immunosorbent assay}

Secreted TNF- $\alpha$ levels were measured in the culture medium by commercial ELISA kits (R\&D systems, UK) according to the manufacturer's instructions.

\section{Immunophenotyping analysis}

CD11b and CD40 marker expressions in BV2 microglial cells induced by LPS were analysed by flow cytometry as described by manufacturer's protocol. The supernatants were discarded after treatment and the cells were washed with warm PBS. Cells were detached using Accutase $^{\mathrm{TM}}$ and centrifuged at $300 \mathrm{xg}$ for $5 \mathrm{~min}$ at $4^{\circ} \mathrm{C}$. After centrifugation, cell pellets were rinsed twice with ice-cold PBS and resuspended $\left(10^{4}\right.$ cells) before being distributed in micro-centrifuge tubes at $100 \mu \mathrm{L}$ each. Blocking was done using 1\% BSA in PBS and incubated at room temperature for $10 \mathrm{~min}$. Subsequently, CD11b (FITC-stained) and CD40 (APC-stained) and their isotype control antibodies were added to respective tubes and incubated in the dark for $20 \mathrm{~min}$ on ice. The cells were rinsed with ice-cold PBS and centrifuged twice for $10 \mathrm{~min}$. On the final wash, $0.5 \mathrm{~mL}$ PBS was added to the cell pellets and kept on ice in the dark. A minimum of 10,000 events of the gated population was acquired from forward and side scatter plots. Dead cells or debris were identified based on lower forward and side scatter signals and eliminated during FACS analysis. Data were analysed using FACS Diva software v6 (BD FACS Canto II, USA).

\section{Western blotting analysis}

After cell treatment, cells were washed with PBS. Cells were dissolved with RIPA buffer + protease inhibitor cocktail. Total protein was measured using Bradford assay kit (Bio-Rad). $20 \mu \mathrm{g}$ of total protein was fractionated by $12 \%$ SDS-polyacrylamide gel electrophoresis (PAGE) and transferred to polyvinylidene difluoride (PVDF) membranes. Blocking of membranes was carried out for $1 \mathrm{hr}$ with skimmed milk (1\%) in TBST, then immunoblotted with conjugated antibodies against MyD88, NF- $x$ B p65, phospho-p38 (Thr180/ Tyr182), phospho-SAPK/JNK (Thr183/Tyr185), phospho-p44/42 (Erk1/2) (Thr202/Tyr204) [PVDF membrane; diluted $1: 1000$ in BSA $(5 \% \mathrm{w} / \mathrm{v})$, TBS $(1 \mathrm{X})$, Tween-20 (1\%)], iNOS [NC membrane; dilution 1:700 in BSA (5\% w/v), TBS (1X), Tween-20 (0.1\%)], $\beta$-actin [dilution 1:3000 in BSA (5\% w/v), TBS (1X), Tween-20 (0.1\%)] and IgG (anti-rabbit)-HRP [1:3000 dilution in skim milk (5\% w/v), TBS (1X), Tween-20 $(0.1 \%)]$. ECL substrate kit, Western Bright ${ }^{\mathrm{TM}}$ enhanced chemiluminescent (Advansta Corporation, San Jose, CA, USA) was used to visualize the immunoblotted protein bands complying with manufacturer's instructions. Protein expression was measured by using Image $\mathrm{Lab}^{\mathrm{TM}}$ software v5.2.1 (Gel Doc ${ }^{\mathrm{TM}} \mathrm{XR}+$, Bio-Rad, CA, USA). All antibodies were from Cell Signalling Technology, MA, USA.

\section{Statistical Analysis}

Data were expressed as mean and standard error of mean (SEM) obtained from repeated experiments. All experiments were repeated thrice with $n=3$. Group differences were statistically assessed by one-way analysis of variance (ANOVA) and Dunnett's test was applied as a post hoc test. Statistical significant were considered if $p<0.05$. Prism 5 (GraphPad Software, San Diego, CA, USA) was used for statistical analysis.

\section{RESULTS \\ Cell viability}

To show that EA was not toxic to BV2 microglial cells, MT'T was applied to measure the cell viability following treatment with EA and LPS. Figure 1a depicts a no toxic effect of EA at concentrations of 0.5 to $10 \mu \mathrm{M}$.

\section{Ellagic acid attenuated NO production and iNOS protein expression}

iNOS is an inflammation-related enzyme needed in NO production. It was found that EA significantly $(P<0.001)$ attenuated NO development dose-dependently in LPSinduced BV2 cells (Figure 1b). Furthermore, using Western blot, the study found that EA (5 and $10 \mu \mathrm{M}$ ) significantly reduced iNOS in comparison to LPS alone (Figure 1c).

\section{Ellagic acid inhibited LPS-induced production of TNFa}

TNF $\alpha$ is a pro-inflammatory cytokine. The study investigated the effects of LPS stimulation on the activation of BV2 cells and whether EA affected the production of $T N F \alpha$. Figure 2 shows that EA inhibited TNF $\alpha$ production dose-dependently in LPS-stimulated BV2 cells as determined by ELISA.

\section{Ellagic acid inhibited NF-kB signalling pathway via MyD88}

Activation of $\mathrm{MyD} 88$ and $\mathrm{NF}-x \mathrm{~B}$ are involved in controlling inflammatory response and modulate the development of pro-inflammatory cytokines. ${ }^{18}$ The investigation into the effects of EA on NF- $x \mathrm{~B}$ inflammatory pathway via MyD88 in LPS-stimulated BV2 microglial cells revealed in Figure 3 where LPSinduced $\mathrm{NF}-x \mathrm{~B}$ p65 via MyD88 was significantly $(p<0.001)$ decreased by EA in a concentration dependent manner as determined by Western blot. 

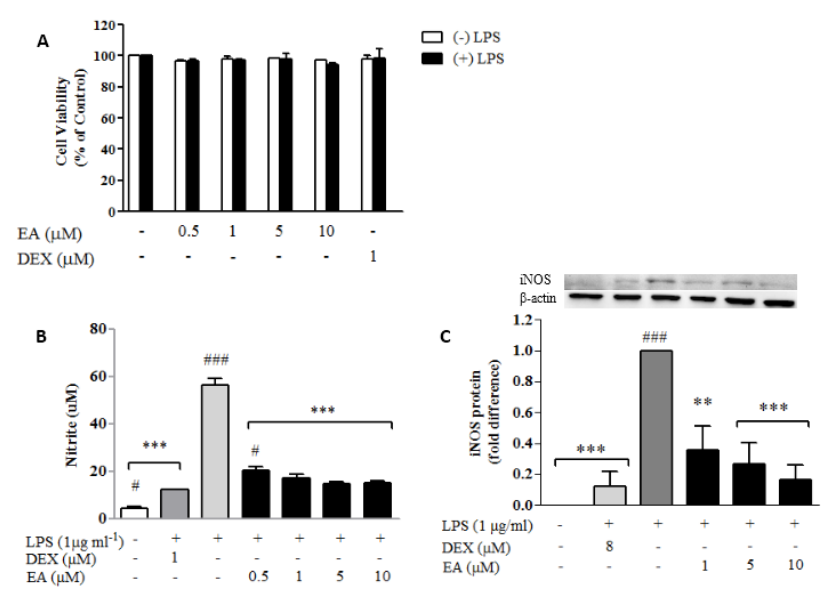

Figure 1: Effects of ellagic acid (EA) on cell viability, production of NO and iNOS protein expression on LPS-induced BV2 cells. Cultures were pre-treated with various doses of EA for $24 \mathrm{hr}$ and then stimulated with LPS (1 $\left.\mu \mathrm{g} \mathrm{mL}^{-1}\right)$ for $24 \mathrm{hr}$. (a) MTT assay. (b) Griess reaction. (c) Western blot. Blots are representative blots. The relative ratio of (c) iNOS/ $\beta$ actin is shown. Data represent mean \pm SEM of three independent experiment ${ }^{*}$ : $p<0.05 ;{ }^{* *}: p<0.01 ;{ }^{* *}: p<0.001$ vs dexamethasone (DEX) \#: $p<0.05 ; \# \#: p<0.01$ and \#\#\#: $p<0.001)$.

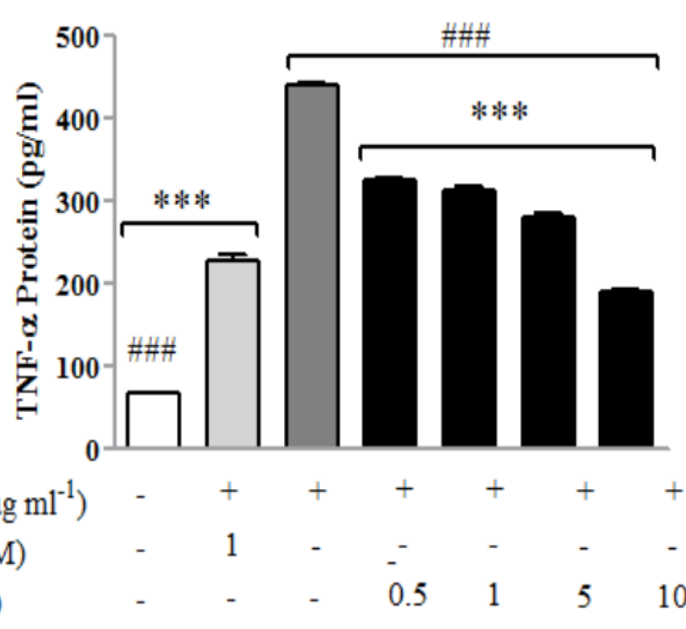

Figure 2: Effect of ellagic acid (EA) on the production of TNFa by LPS activated BV2 cells. Cultures were pre-treated with EA for $24 \mathrm{hr}$, followed by LPS stimulation for $24 \mathrm{hrs}$. The TNFa expression in the culture supernatant was analysed using ELISA. The expression of TNFa in the cell culture supernatant was analysed using ELISA. Data represented mean \pm SEM of three independent experiments. One-way ANOVA and Dunnett's multiple comparison's test were used to analysed data (*: $p<0.05$; ${ }^{* *}: p<0.01$; ${ }^{* * *}: p<0.001$ vs dexamethasone (DEX) \#: $p<0.05$; \#\#: $p<0.01$ and \#\#\#: $p<0.001)$.

\section{Ellagic acid suppresses the phosphorylation of MAPKs}

MAPKs (P38 MAPK, JNK and ERK) phosphorylate various range transcription factors which modulate and regulate inflammatory response by producing pro-inflammatory mediators and cytokines. ${ }^{19}$ Figure

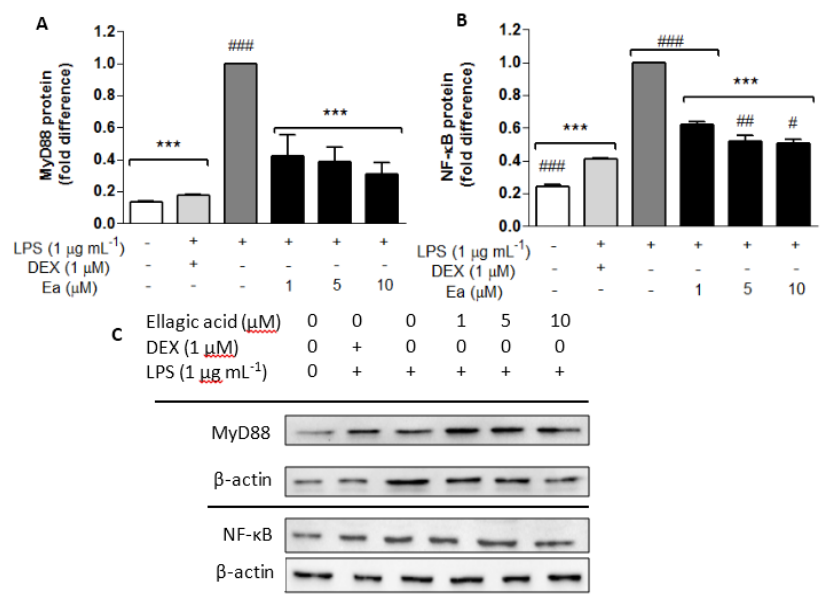

Figure 3: Effect of ellagic acid (EA) on NF-KB via MyD88 signalling pathway by LPS-activated BV2 cells. Cultures were pre-treated with EA for $24 \mathrm{hr}$, followed by LPS $\left(1 \mu \mathrm{g} \mathrm{mL}^{-1}\right)$ for $24 \mathrm{hr}$. a, b Quantification was performed on three independent experiments and are presented as mean \pm SEM. $c$ Western blot analysis of MyD88 and NF-kB. Blots are representative of a and b. One-way ANOVA and Dunnett's multiple comparison's test were used to analysed data $\left(^{*}: p<0.05 ;{ }^{* *}: p<0.01\right.$; ${ }^{* * *}$ : $p<0.001$ vs dexamethasone (DEX) \#: $p<0.05$; \#\#: $p<0.01$ and \#\#\#: $p<0.001)$.

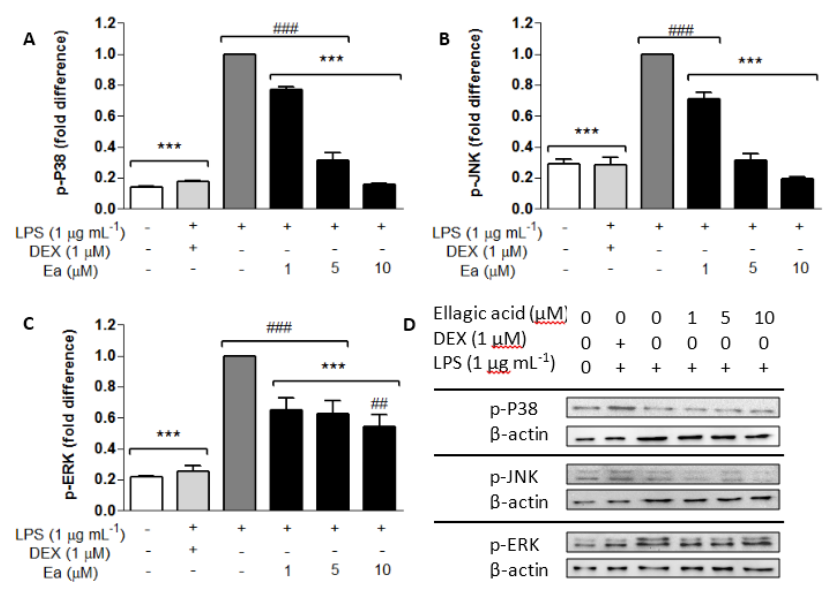

Figure 4: Effects of ellagic acid (EA) on MAPK signalling pathway by LPS-activated BV2 cells. Cells were incubated with various concentration of EA for $24 \mathrm{hr}$ and then treated with LPS ( $\left.1 \mu \mathrm{g} \mathrm{mL}^{-1}\right)$ for $24 \mathrm{hr}$. a, b, c Quantitation was performed on three independent experiments and are presented as mean \pm SEM. One-way ANOVA and Dunnett's multiple comparison's test were used to analysed data. EA *: $p<0.05$; ${ }^{* *}: p<0.01$; ***: $p<0.001$ vs dexamethasone (DEX) \#: $p<0.05$; \#\#: $p<0.01$ and \#\#: $p<0.001$. d Western blot analysis of phospho-P38, phospho-JNK and phospho-ERK. Blots are representative of $a, b$ and $c$.

4 indicates a large increase in P38 MAPK, ERK and JNK phosphorylation following stimulation by LPS. Pre-treatment with EA significantly reduced $(p<0.001)$ phosphorylation of P38 MAPK, ERK and JNK at $1 \mu \mathrm{M}, 5 \mu \mathrm{M}$ and $10 \mu \mathrm{M}$ concentrations (Figure 4a, b, c, respectively). 


\section{Ellagic acid modulates microglial inflammatory phenotype}

Upon LPS stimulation, a characteristic of inflammatory of microglial phenotype was seen on the cell surface where costimulatory molecules (CD40) and key adhesion (CD11b) were expressed. LPS-induced BV2 microglial cells significantly increased surface expression of CD40 and CD11b (Figure 5a and b). Pre-treatment with EA at various concentrations $(1,5$ and $10 \mu \mathrm{M})$ significantly reduced $(\phi<0.001)$ CD11b by $50 \%$ (Figure $5 \mathrm{a}$ ) which was seen across all three concentrations, whereas the reduction of CD40 was also significant $(p<0.001)$ at EA concentrations of $5 \mu \mathrm{M}$ and $10 \mu \mathrm{M}$.

\section{DISCUSSION AND CONCLUSION}

Polyphenol compounds such as ellagic acid (EA) are found abundantly in nuts and fruits such as raspberries and pomegranates. ${ }^{13}$ To the best of our knowledge, this is the first evidence indicating the protective effects of EA in suppressing LPS-stimulated acute neuroinflammation in murine BV2 microglia.

Pre-treatment of EA $\left(0.5-10 \mu \mathrm{g} \mathrm{mL}^{-1}\right)$ significantly diminished neuroinflammation induced by LPS in a dose-

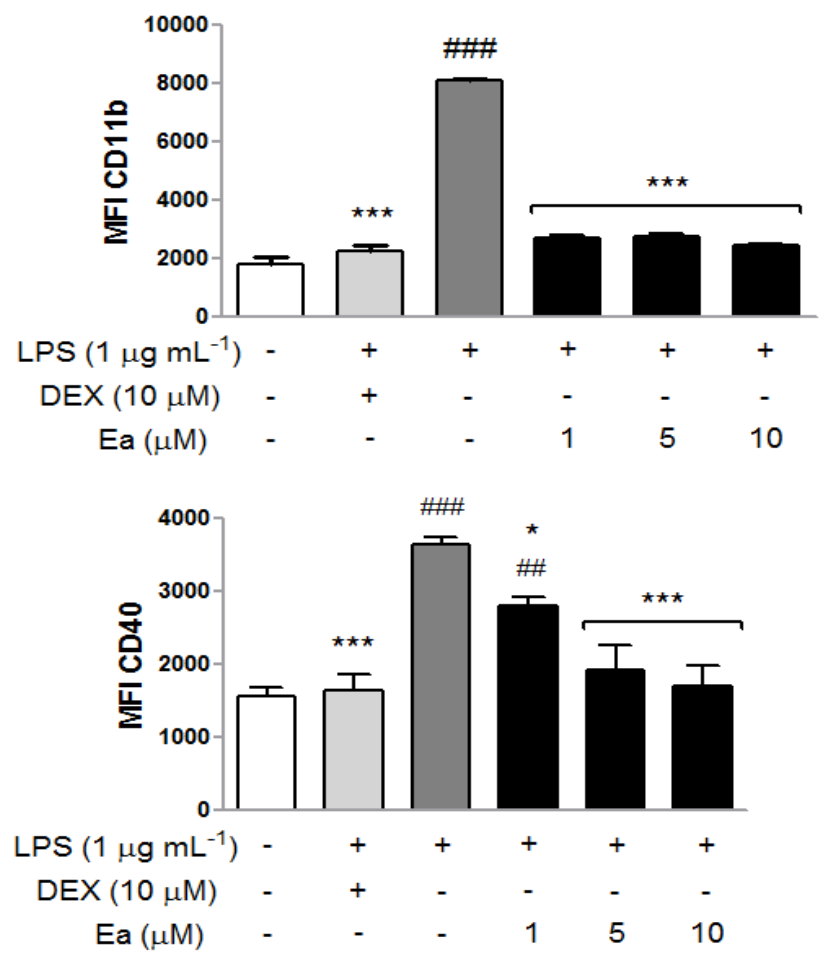

Figure 5: Ellagic acid (EA) modulates inflammatory cell surface marker expression in LPS-treated BV2 microglia. Flow cytometric analysis of EA-treated BV2 cells following LPS activation. $a$ and $b$ quantitation of $C D 11 b$ and CD40 expression by median fluorescence intensity (MFI) from three independent experiments. Data are expressed as mean \pm SEM where EA $\left({ }^{*} p<0.05\right)$ vs dexamethasone (DEX) $(\# p<0.05)$. dependent manner. The protective capacity of EA as revealed in this study has attenuated neuroinflammation in the extend of reduction of murine microglial activity which was accompanied by a decrease in the cellular network of inflammatory signalling and a decrease in the relative mediator/pro-inflammatory cytokines.

This study was aimed to examine LPS-mediated activation of BV2 cells in relevance to the putative protective effects of EA. This work included the use of murine BV2 microglial cells and triggered an inflammatory reaction to LPS. Previous studies have shown that LPS-activated microglia secrete various proinflammatory mediators and cytokines. ${ }^{20}$ In response to LPS stimuli, activated BV2 microglia promote the production of NO and TNF $\alpha$. Such neuroinflammatory symptoms cause neuronal damage and lead to neurodegenerative diseases. ${ }^{21}$ There is evidence that iNOS/NO production differs from single-cell culture in astrocyte-microglial mixed culture conditions. ${ }^{22}$ Numerous observations have also been made on the possible effects of NO production in brain cells, especially in co-culture conditions such as astrocytes and microglia. ${ }^{23}$ In this analysis, the up-regulation of iNOS by LPS stimulation has been shown to lead to the development of NO and has the potential to increase $\mathrm{NF}-x \mathrm{~B}$ activation as demonstrated in a report by McAdam and co-workers. ${ }^{24}$ Although our study did not correlate between iNOS/NO and NF- $x \mathrm{~B}$ activation, we demonstrated that EA pre-treatment decreased iNOS/NO and NF- $x \mathrm{~B}$ p 65 subunits in BV2 microglial cells mediated by LPS. In addition, the NO produced also activated not only NF- $x \mathrm{~B}$ but also MAPKs. ${ }^{25}$ Our investigation on the protective effects of EA was continued with determining the inflammatory factors produced in activated BV2 cells mediated by LPS. The results showed that EA pre-treatment significantly inhibited microglial activation (CD11b and CD40) and the development of TNF $\alpha$ stimulated by LPS. These results demonstrate the potential therapeutic effects of $\mathrm{EA}$ in various inflammatory reactions.

The increased expression of CD11b is an indicator of microglial activation. According to Roy and colleagues ${ }^{26}$ the expression of CD11b starts after NO development and the signalling mechanisms for CD11b expression in LPS-induced microglia are not fully understood. In this analysis, EA dose-dependently decreased CD11b signals.

CD40 expression in microglial cells protects the CNS from invasion and retention of inflammatory leukocytes. ${ }^{27}$ CD40 and microglial activation are involved in various CNS diseases such as multiple sclerosis and Alzheimer's disease and therefore, we 
were specifically investigating the protective role of EA on CD40 expression in LPS-mediated BV2 activation. According to Qin and colleagues, CD40 expression upon LPS activation in BV2 microglial cells is an intricate process requiring endogenous production of IFN- $\beta$ and STAT- $1 \alpha$ activation. Also, LPS induction of CD40 expression involves NF- $x \mathrm{~B}$ elements in the CD40 promoter. ${ }^{28}$ Therefore the results of our present study are in agreement with Qin and colleagues in which LPS activated NF- $x \mathrm{~B} / \mathrm{p} 65$ subunit contributing to the binding of CD40 promoter which eventually activated the CD40 expression on the surface of microglial cells. Dysregulated activation of microglial cells can trigger a phenotype shift leading to disruption of signalling pathways and inflammatory process, ${ }^{13}$ chronic mediator/ pro-inflammatory cytokine secretion and neuronal injury/death seen in neurodegenerative diseases. ${ }^{29}$ The three inflammatory signalling pathways include MAPKs, $\mathrm{P} 13 \mathrm{~K} / \mathrm{Akt}$ and $\mathrm{NK}-x \mathrm{~B} \cdot{ }^{30}$ In mammalian cells, MAPKs consist of extracellular signal protein (p42/44; ERK), the p38 MAPK and the $\mathrm{JNK}^{31}$ which are expressed in many tissues, including CNS. ${ }^{32}$ The activation of intracellular signalling includes the phosphorylation of these MAPKs. There is currently, insufficient knowledge on the expression of MAPK in the brain due to LPS or bacterial infection. ${ }^{32}$ Our findings showed that LPS increased the phosphorylated p38, JNK and ERK1/2 in the control group after $72 \mathrm{hrs}$ and this phosphorylation was significantly reduced by EA pre-treatment in BV2 microglial cells. Limited findings have shown that phosphorylation of p38 MAPK, ERK1/2 and JNK occurred in response to LPS in cells pre-treated with corylin ${ }^{33}$ and benfiotamine, ${ }^{34}$ thus MAPKs signalling may be involved in $\mathrm{TNF} \alpha$ production in microglia.

Transcription factor $\mathrm{NF}-\varkappa \mathrm{B}$ omnipresently regulates the gene expression of a broad variety of neuropathological processes, including hypoxia, neuroinflammation and trauma. ${ }^{35} \mathrm{~A}$ more important function for fully activated $\mathrm{NF}-x \mathrm{~B}$ was also played by the subunit NF- $x \mathrm{~B} / \mathrm{p} 65$ and our analysis showed that LPS induced NF- $x \mathrm{~B} /$ p65 phosphorylation after 72 hrs. Pre-treatment with EA not only decreased the NF- $x \mathrm{~B} / \mathrm{p} 65$ levels but also the downstream pro-inflammatory cytokine TNF $\alpha$ levels. Previous study found that inhibition of MAPKs decreased the upregulation of $\mathrm{NF}-x \mathrm{~B} / \mathrm{p} 65$ following treatment with LPS, involving the downstream molecules of NF-xB and MAPKs and there was a cross link between them. ${ }^{32}$

This work thus identified the effects of EA in LPSinduced expression of MyD88 in BV2 cells. The adaptor protein MyD88 mediates the signals for most TLRs leading to activation of MAPKs and NF- $x \mathrm{~B}$, which results in the formation of inflammatory mediators. ${ }^{25}$ The results in this study demonstrated that EA blocked MyD88 expression induced by LPS suggestive of an anti-neuroinflammatory effect of EA by modulating the interaction of MyD88 with its downstream counterparts. Consequently, these results also supported the putative role of EA in preventing neuroinflammation but its application in prevention of neurodegenerative diseases requires further investigations.

\section{ACKNOWLEDGEMENT}

This work is supported by The Ministry of Agriculture, Malaysia (Grant number NH1024D023). We would like to thank Associate Professor Dr. Sharmili Vidyadaran for her generosity in providing the BV2 microglial cells.

\section{CONFLICT OF INTEREST}

The authors declare no conflict of interest.

\section{ABBREVIATIONS}

Akt: Protein kinase B; APC: Allophycocyanin; BSA: Bovine serum albumin; CD: Cluster of difference; CNS: Central nervous system; $\mathbf{C O}_{2}$ : Carbon dioxide; COX-2: Cyclooxygenase-2; DMSO: dimethyl sulfoxide; EA: Ellagic acid; ERK: Extracellular signal-regulated kinases; FITC: Fluorescein-5-isothiocyanate; hrs: hour; HRP: Horse radish peroxidase; IL: Interleukin; iNOS: Inducible nitric oxide synthase; JNK: C-JUN N-terminal kinases; LPS: Lipopolysaccharide; MAPKs: Mitogen-activated protein kinase; $\mathbf{m g} \mathbf{~ m L}^{-1}$ : Miligram per mililitre; min: Minute; mL: Mililitre; MTT: 3-(4,5-dimethylthiazol-2-yl)-2,5-diphenyltetrazolium bromide; MyD88: Myeloid differentiation primary response 88; NC: Nitrocelullose; NF- $\boldsymbol{x B}$ : Nuclear factor kappa B; nm: Nanometre; NO: Nitric oxide; NSAIDs: Non-steroidal anti-inflammatory drugs; P13K: phosphatidylinositol 3-kinase; p38: P38 mitogenactivated protein kinases; PBS: Phosphor buffer saline; PVDF: Polivinylidenedifluoride; RT: Room temperature; TBS: Tris buffered saline; TBST: tris buffered saline with tween-20; TNF $\alpha$ : Tumour necrosis factor alpha; $\mathbf{U ~ m L}^{-1}: \mu \mathrm{g} \mathrm{mL}^{-1} ; \mu \mathbf{M}$ : micromolar.

\section{REFERENCES}

1. Carniglia L, Ramírez D, Durand D, Saba J, Turati J, Caruso C, et al. Neuropeptides and Microglial Activation in Inflammation, Pain and Neurodegenerative Diseases. Mediators Inflamm. 2017.

2. Wang WY, Tan MS, Yu JT, Tan L. Role of pro-inflammatory cytokines released from microglia in Alzheimer's disease. Ann Transl Med. 2015;3(10):136. Available from: http://www.ncbi.nlm.nih.gov/pubmed/26207229 
3. Jayathirtha MG, Mishra SH. Preliminary immunomodulatory activities of methanol extracts of Eclipta alba and Centella asiatica. Phytomedicine. 2004;11(4):361-5. Available from: http://www.sciencedirect.com/science/ article/pii/S0944711304703414

4. Prinz M, Priller J. Microglia and brain macrophages in the molecular age : from origin to neuropsychiatric disease. Nat Publ Gr. 2014;15(5):300-12. Available from: http://dx.doi.org/10.1038/nrn3722

5. Ransohoff RM, Cardona AE. The myeloid cells of the central nervous system parenchyma. Nature. 2010;468(7321):253-62. Available from: http://www. nature.com/doifinder/10.1038/nature09615

6. Lull ME, Block ML. Microglial Activation and Chronic Neurodegeneration. Neurotherapeutics. 2011;7(4):354-65.

7. Sochocka M, Diniz BS, Leszek J. Inflammatory Response in the CNS: Friend or Foe?. Mol Neurobiol. 2017;54(10):8071-89. Available from: https://www. ncbi.nlm.nih.gov/pmc/articles/PMC5684251/pdf/12035_2016_Article_297. pdf

8. Amor S, Peferoen LAN, Vogel DYS, Breur M, Valk PV, Baker D, et al. Inflammation in neurodegenerative diseases: An update. Immunology. 2014;142(2):151-66.

9. Mottahedin A, Ardalan M, Chumak T, Riebe I, Ek J, Mallard C, et al. Effect of Neuroinflammation on Synaptic Organization and Function in the Developing Brain: Implications for Neurodevelopmental and Neurodegenerative Disorders. Frontiers in Cellular Neuroscience. 2017;11:190. [cited 2019 Mar 4]; Available from: www.frontiersin.org

10. Jha MK, Jeon S, Suk K. Glia as a Link between Neuroinflammation and Neuropathic Pain. Immune Netw. 2012;12(2):41. Available from: http://www. ksimm.or.krvolume12number

11. Farbood Y, Sarkaki A, Dolatshahi M, Taqhi MSM, Khodadadi A. Ellagic Acid Protects the Brain Against 6-Hydroxydopamine Induced Neuroinflammation in a Rat Model of Parkinson's Disease. Basic Clin Neurosci. 2015;6(2):83-9. Available from: http://www.ncbi.nlm.nih.gov/pubmed/27307952

12. Harikrishnan H, Jantan I, Haque MA, Kumolosasi E. Phyllanthin from Phyllanthus amarus inhibits LPS-induced proinflammatory responses in U937 macrophages via down regulation of NF-KB/MAPK/PI3K-Akt signaling pathways. Phyther Res. 2018;32(12):2510-9. [cited 2018 Oct 22]; Available from: http://doi.wiley.com/10.1002/ptr.6190

13. Rosillo MA, Sanchez-Hidalgo M, Cárdeno A, DeLastra CA. Protective effect of ellagic acid, a natural polyphenolic compound, in a murine model of Crohn's disease. Biochem Pharmacol. 2011;82(7):737-45. [cited 2018 Nov 17] Available from: http://www.ncbi.nlm.nih.gov/pubmed/21763290

14. Chao PC, Hsu CC, Yin MC. Anti-inflammatory and anti-coagulatory activities of caffeic acid and ellagic acid in cardiac tissue of diabetic mice. Nutr Metab. 2009;6(1):33.

15. Corbett S, Daniel J, Drayton R, Field M, Steinhardt R, Garrett N. Evaluation of the anti-inflammatory effects of ellagic acid. J Perianesthesia Nurs. 2010;25(4):214-20.

16. Gopalakrishnan L, Ramana LN, Sethuraman S, Krishnan UM. Ellagic acid encapsulated chitosan nanoparticles as anti-hemorrhagic agent. Carbohydr Polym. 2014;111:215-21.

17. Ibrahim S, Mohamed A, Jantan I, Nafiah MA, Seyed MA. Dendritic cells pulsed with generated tumor cell lysate from Phyllanthus amarus immune response. BMC Compliment Altern Med. 2018;18(1):1-14.

18. Zou J, Shankar N. Roles of TLR/MyD88/MAPK/NF-KB Signaling Pathways in the Regulation of Phagocytosis and Proinflammatory Cytokine Expression in Response to E. faecalis Infection. PLoS One. 2015;10(8):e0136947. Available from: https://www.ncbi.nlm.nih.gov/pmc/articles/PMC4552673/pdf/ pone.0136947.pdf

19. Cargnello M, Roux PP. Activation and function of the MAPKs and their substrates, the MAPK-activated protein kinases. Microbiol Mol Biol Rev [Internet]. 2011 Mar 1 [cited 2018 Oct 19];75(1):50-83. Available from: http:// www.ncbi.nlm.nih.gov/pubmed/21372320
20. Saijo K, Glass CK. Microglial cell origin and phenotypes in health and disease. Nat Rev Immunol. 2011;11(11):775-87. Available from: http://dx.doi. org/10.1038/nri3086

21. Amor S, Puentes F, Baker D, Der VPV. Inflammation in neurodegenerative diseases. Immunology. 2010;129(2):154-69.

22. Solà C, Casal C, Tusell JM, Serratosa J. Astrocytes enhance lipopolysaccharide-induced nitric oxide production by microglial cells. Eur J Neurosci. 2002;16(7):1275-83.

23. Kim YJ, Hwang SY, Oh ES, Oh S, Han IO. IL-1 $1 \beta$, an immediate early protein secreted by activated microglia, induces iNOS/NO in C6 astrocytoma cells through p38 MAPK and NF-kB pathways. J Neurosci Res. 2006;84(5):103746. Available from: http://doi.wiley.com/10.1002/jnr.21011

24. McAdam E, Haboubi HN, Forrester G, Eltahir Z, Spencer-Harty S, Davies C, et al. Inducible Nitric Oxide Synthase (iNOS) and Nitric Oxide (NO) are Important Mediators of Reflux-induced Cell Signalling in Esophageal Cells. Carcinogenesis. 2012;33(11):2035-43. [cited 2019 Mar 20]Available from: https://academic.oup.com/carcin/article-lookup/doi/10.1093/carcin/bgs241

25. Lim HS, Kim YJ, Kim BY, Park G, Jeong SJ. The anti-neuroinflammatory activity of tectorigenin pretreatment via down regulated NF-KB and ERK/ JNK pathways in BV-2 microglial and microglia inactivation in mice with lipopolysaccharide. Front Pharmacol. 2018. [cited 2018 Nov 9]. Available from: www.frontiersin.org

26. Roy A, Fung YK, Liu X, Pahan K. Up-regulation of Microglial CD11b Expression by Nitric Oxide. J Biol Chem. 2006;281(21):14971-80. Available from: http://www.jbc.org/lookup/doi/10.1074/jbc.M600236200

27. Laman JD, Meurs MV, Schellekens MM, DeBoer M, Melchers B, Massacesi $\mathrm{L}$, et al. Expression of accessory molecules and cytokines in acute EAE in marmoset monkeys (Callithrix jacchus). J Neuroimmunol. 1998;86(1):30-45.

28. Qin H, Wilson CA, Lee SJ, Zhao X, Benveniste EN. LPS induces CD40 gene expression through the activation of NF-kappaB and STAT-1alpha in macrophages and microglia. Blood. 2005;106(9):3114-22. Available from: http://www.bloodjournal.org/cgi/doi/10.1182/blood-2005-02-0759

29. Lehnardt S. Innate Immunity and Neuroinflammation in the CNS: The Role of Microglia in Toll-Like Receptor-Mediated Neuronal Injury. Gila. 2010;58(3):253-63.

30. Zhang L, Zhang J, Jiang X, Yang L, Zhang Q, Wang B, et al. Hydroxytyrosol Inhibits LPS-Induced Neuroinflammatory Responses via Suppression of TLR-4-Mediated NF-KB P65 Activation and ERK Signaling Pathway. Neuroscience. 2020;426(1):189-200. Available from: http://dx.doi. org/10.1016/j.neulet.2010.12.019

31. Dong C, Davis RJ, Flavell RA. MAP kinases in the immune response. Annu Rev Immunol. 2002;20(1):55-72.

32. Zhu X, Wei D, Chen O, Zhang Z, Xue J, Huang S, et al. Up regulation of CCL3/MIP-1alpha regulated by MAPKs and NF-kappaB mediates microglial inflammatory response in LPS-induced brain injury. Acta Neurobiol Exp. 2016;76(4):304-17. [cited 2020 Jan 23] Available from: http://www.ncbi.nlm. nih.gov/pubmed/28094821

33. Huang MY, Tu CE, Wang SC, Hung YL, Su CC, Fang SH, et al. Corylin inhibits LPS-induced inflammatory response and attenuates the activation of NLRP3 inflammasome in microglia. BMC Complement Altern Med. 2018;18(1):221. Available from: https://bmccomplementalternmed.biomedcentral.com/ articles/10.1186/s12906-018-2287-5

34. Bozic I, Savic D, Laketa D, Bjelobaba I, Milenkovic I, Pekovic S, et al. Benfotiamine attenuates inflammatory response in LPS stimulated BV-2 microglia. PLoS One. 2015;10(2):1-23. Available from: http://dx.doi. org/10.1371/journal.pone.0118372

35. Nurmi A, Vartiainen N, Pihlaja R, Goldsteins G, Yrjänheikki J, Koistinaho J. Pyrrolidine dithiocarbamate inhibits translocation of nuclear factor kappa-B in neurons and protects against brain ischaemia with a wide therapeutic time window. J Neurochem. 2004;91(3):755-65. 


\section{PICTORIAL ABSTRACT}

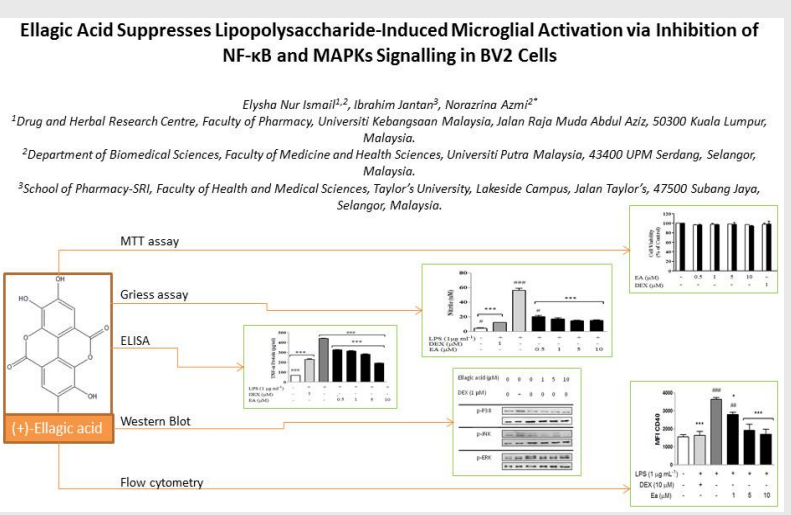

\section{SUMMARY}

Dysregulated activation of microglia is one of the hallmarks of neurodegenerative diseases that results in neuroinflammation. Neurodegenerative disorders such as Parkinson's disease and Alzheimer's disease have been associated with neuroinflammation. The present study proposed a protective role of ellagic acid (EA) against neuroinflammation studied using LPS-activated BV2 microglial cells. The results provide an insight into the mechanisms of EA in preventing activation of microglia mediated through attenuation of inflammatory signalling molecules such as MAPKs and NF- $\kappa \beta$. However, the putative role of EA in neuroprotection and its application in prevention of neurodegenerative diseases require further investigations.

\section{About Authors}

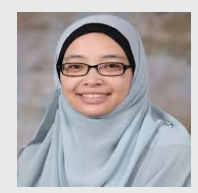

Elysha Nur Ismail, Lecturer in Immunology, Department of Biomedical Sciences, Faculty of Medicine and Health Sciences, Universiti Putra Malaysia, Selangor, Malaysia.

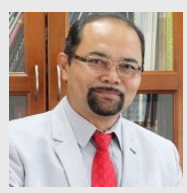

Ibrahim Jantan, Honorary Professor, Institute of Systems Biology (INBIOSIS), Universiti Kebangsaan Malaysia, UKM Bangi, Selangor, Malaysia.

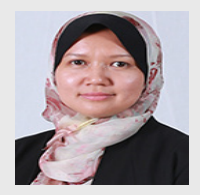

Norazrina Azmi, Associate Professor in Neuropharmacology, Drug and Herbal Research Centre, Faculty of Pharmacy, Universiti Kebangsaan Malaysia, Jalan Raja Muda Abdul Aziz, Kuala Lumpur, Malaysia.

Cite this article: Ismail EN, Azmi N, Jantan I. Ellagic Acid Protects Against Activation of Microglia by Inhibiting MAPKs and NF-kB Signalling. Indian J of Pharmaceutical Education and Research. 2020;54(3s):s529-s536. 